\title{
Thermalisation properties of various field theories
}

\section{Marietta M. Homor* and Antal Jakovac}

Eotvos Lorand University

E-mail: homor.marietta.megmail.com, jakovacecaesar.elte.hu

\begin{abstract}
Motivated by the hadronization properties of heavy-ion collisions, we study the thermalisation properties of field theories. First of all, we present our recent observations regarding the local energy-density and momentum distributions in the classical $\Phi^{4}$ theory. As this theory exhibits interesting features regarding these questions, we continue the research by the MC simulation of the SU(3) Yang-Mills theory and we intend to investigate further in more complicated theories.
\end{abstract}

34th annual International Symposium on Lattice Field Theory

24-30 July 2016

University of Southampton, UK

${ }^{*}$ Speaker. 


\section{Introduction}

It is well-known from experiments, that the matter formed in relativistic heavy-ion collisions behave as a nearly ideal liquid and the shear-viscosity $(\eta)$ over entropy-density $(s)$ ratio is extremely small [1]. In a liquid-like matter where information is spread by diffusion and the transport coefficient so small, interactions can no longer be neglected. On one hand, strong interactions make difficult to describe hadronization processes, on the other hand, interesting phenomena may occur.

One of such curiosities is related to the transvers momentum $\left(p_{T}\right)$ histograms. One may anticipate that the $p_{t}$ distribution of particle yields is Boltzmannian. However, it seems not to be correct in p-p collisions according to ALICE, CMS, STAR and PHENIX collaborations, respectively [2][3][4][5]. Instead of Boltzmannian, the Tsallis-distribution is proposed.

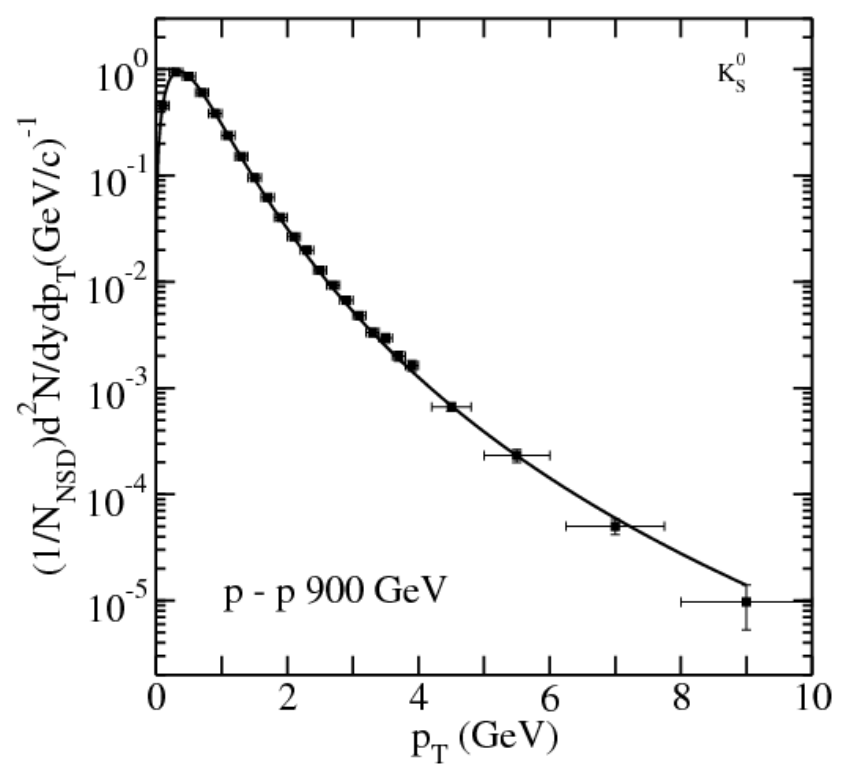

Figure 1: Transverse momentum distribution for $K_{S}^{0}$ as measured by the CMS collaboration [7]. The $0.9 \mathrm{TeV}$ data is compared to the Tsallis-B distribution (continuous full line) by J. Cleymans and D. Worku in [6].

What can be the reason of the discrepancy between the expected and measured distribution functions? It can be of statistical origin: if hadron multiplicity fluctuates according to negativebinomial distribution, then the $p_{T}$ distribution is Tsallis-like even if the event-by event distribution is Boltzmannian [8].

Hunting for a microscopic mechanism, we should understand the radiation of a strongly interacting plasma. If hadrons are created locally, then the creation probability must depend on the local energy-density. This is a measurable quantity, since one can extract the local energy-density distribution from computer simulations, thus we may have a tool to determine the hadron distribution function. We put the concept into action with various toy models, namely the SU(3) Yang-Mills theory and the classical $\Phi^{4}$ theory.

\section{Local energy-density distribution}

As stated in the introduction, our aim is to determine the local energy-density distribution. In 
this section we recall some basic definitions of probability theory.

First of all, let $X$ be a stochastic variable. The indicator of $X$ being in the $[x, x+\Delta x]$ interval is $\mathbb{I}_{[x, x+\Delta x]}(X)$. The expectation value of this indicator equals the probability of $X$ being in the interval:

$$
\left\langle\mathbb{I}_{[x, x+\Delta x]}(X)\right\rangle=\mathscr{P}(X \in[x, x+\Delta x]) .
$$

Let $f(x)$ note the probability density function of $X$. By definition:

$$
f(x)=\lim _{\Delta x \rightarrow 0+} \frac{\mathscr{P}(X \in[x, x+\Delta x])}{\Delta x} .
$$

If we write (2.1) into the definition (2.2) of $f(x)$, then we get a Dirac- $\delta$ approximation and we can write rather formally:

$$
f(x)=\langle\delta(X-x)\rangle
$$

In this work, the quantity in question - in other words the stochastic variable - is the local energy density $\varepsilon_{x}$ where $x$ is an arbitrary space-time coordinate. With this (2.3) becomes:

$$
\mathscr{P}(\varepsilon)=\left\langle\delta\left(\varepsilon_{x}-\varepsilon\right)\right\rangle
$$

However, (2.4) is sometimes confused by a different energy-distribution. Let $A(\Phi(t), \Pi(t))$ be a local physical quantity, where $\Phi(t)$ and $\Pi(t)$ are some field and momentum field variables depending on time $t$. The ensemble average of $A$ is the following:

$$
\langle A(\Phi(t), \Pi(t))\rangle=\int \mathscr{D} \bar{\Phi} \mathscr{D} \bar{\Pi} A(\bar{\Phi}, \bar{\Pi}) f(\bar{\Phi}, \bar{\Pi}),
$$

where $f(\bar{\Phi}, \bar{\Pi})$ is a probability density function as well. The two distributions can be connected by the following train of thought. If we are interested in the expectation value of whether $\varepsilon_{x}$ takes on a certain value $\varepsilon$, then we can formally substitute $A$ with $\delta\left(\varepsilon_{x}-\varepsilon\right)$ in equation (2.5). Finally, we get:

$$
\left\langle\delta\left(\varepsilon_{x}-\varepsilon\right)\right\rangle=\int \mathscr{D} \bar{\Phi} \mathscr{D} \bar{\Pi} \delta\left(\varepsilon_{x}-\varepsilon\right) f(\bar{\Phi}, \bar{\Pi}) .
$$

The left hand side of the equation (2.6) is the local energy-density distribution which we want to determine from our simulations. On the right hand side, we have the distribution function $f(\bar{\Phi}, \bar{\Pi})$ of the ensemble (e.g. Boltzmannian for canonical ensemble), which is dependent on the total energy of the system through the field variables $\Phi$ and $\Pi$.

The main point is that these two distributions are not necessarily the same, although they sometimes coincide. As a result, it is possible in a Monte Carlo simulation, that the local energydensity distribution is not Boltzmannian while the configurations are still generated by Boltzmanndistribution.

In the following sections, we consider the real time simulation of the classical $\Phi^{4}$ theory in 3 dimensions and perform a standard Monte Carlo simulation with heat-bath algorithm for the Euclidean SU(3) gauge theory. 


\section{A toy model: classical $\Phi^{4}$ theory}

Our first toy modell is the well-known classical $\Phi^{4}$ theory. One of the advantages of classical theories is that we can perform real-time simulations by successively solving the canonical equations and we can calculate physical quantities that are hardly accessible in other methods. One of such examples is the shear-viscosity which is analized in [9].

The energy-density at a certain lattice coordinate of the 3D space $(\mathbf{x})$ is given by the Hamiltonian of the system. The discretized form is the following:

$$
\varepsilon_{\mathbf{x}}=\frac{1}{2} \Pi_{\mathbf{x}}^{2}+\frac{1}{2}(\nabla \Phi)_{\mathbf{x}}^{2}+\frac{m^{2}}{2} \Phi_{\mathbf{x}}^{2}+\frac{\lambda}{24} \Phi_{\mathbf{x}}^{4} .
$$

We note, that the total energy of the continuous theory is constant and our simulation algorithm preserves this property.

The temperature $(T)$ of the system is calculated by the following formula [9]:

$$
T=\frac{1}{2 N^{3}}\left\langle\left|\Pi_{k}\right|^{2}\right\rangle
$$

where $N^{3}$ is the number of lattice sites and $\Pi_{k}$ is the Fourier-transformed momentum field. We use this formula to check whether the system reached thermal equilibrium by verifying that $\left\langle\left|\Pi_{k}\right|^{2}\right\rangle$ is independent of $\mathbf{k}$ (equipartition). It turned out, that we can distinguish two time scales, as higher modes thermalise much faster than low ones. After 10000 time steps, the system can be considered fully thermalised.

\section{Numerical results}

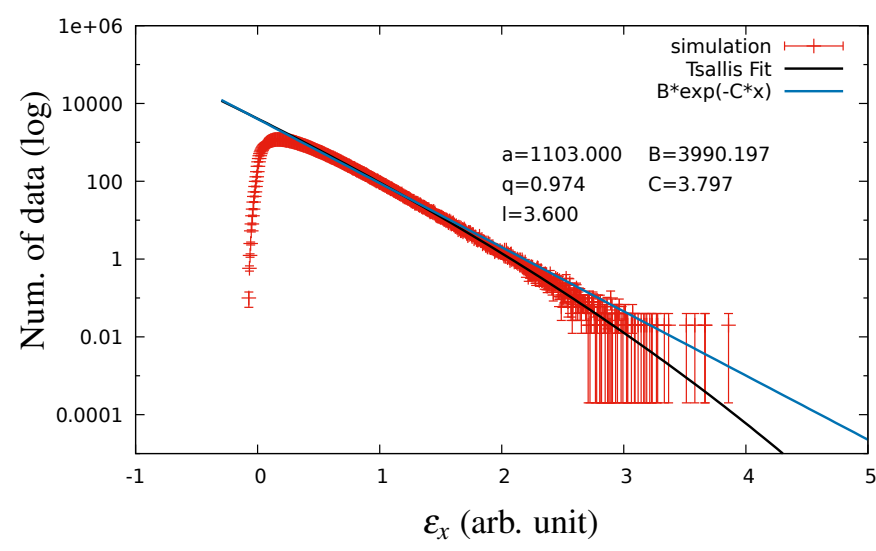

Figure 2: Local energy-density histogram with Boltzmann (blue line) and Tsallis (black line) fits on semi$\log$ scale after 17 time steps. Simulation with random initial condition for $\Pi_{\mathbf{x}}$. Data points are averaged from 50 simulations and shown with their standard error.

The results of our simulations for two different initial conditions on a $50^{3}$ lattice are shown in figures 2 and 3. It is clear, that at the beginning (after 17 time steps) the distribution is not Boltzmannian, so we tried the following function as well:

$$
\mathscr{P}(\varepsilon)=a[1+(q-1) \beta \varepsilon]^{\frac{1}{1-q}} .
$$




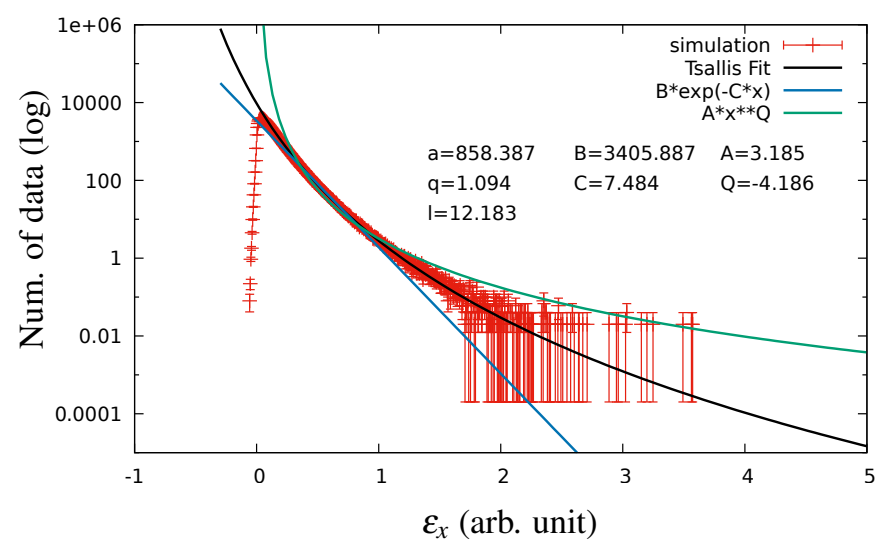

Figure 3: Local energy-density histogram with simple power function (green line), Boltzmann (blue line) and Tsallis (black line) fits on semi-log scale after 17 time steps. Simulation with secant hyperbolic initial condition for $\Pi_{\mathbf{x}}$. Data points are averaged from 50 simulations and shown with their standard error.

It is the so-called Tsallis-distribution. Note that for $q \rightarrow 1$ it gives back the Boltzmann-distribution. It turned out that Tsallis fits well for both cases.

However, in these cases the system is still far from equilibrium. The main question is that whether the Boltzmann-distribution is restored with equilibrium or not, so we continue the analysis for the time dependence of the $q$ parameter on a much longer scale. Based on figure 4, one can conclude, that the $q$ parameter remains consistently over one even after full thermalisation. Moreover, the shape of the distribution does not significantly change after only a few 17-25 time steps. The Tsallis-parameter takes its equilibrium value already in the pre-thermalised state (i.e. when only higher modes are thermalised) within error. The average values on figure 4 are in the order of the experimental values.

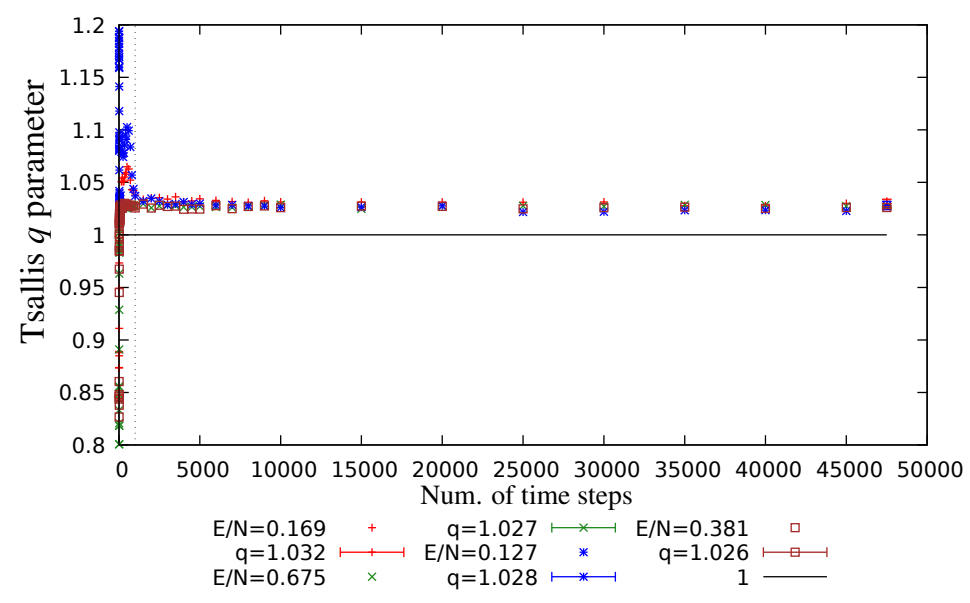

Figure 4: Time dependence of the Tsallis parameter at 4 different total energies with various initial conditions. The last points indicate the average values for each energies from 1000 to 45000 time steps.

As a comparison, we checked the momentum-distribution. Based on the equation (2.5) with the same train of thought as for the energy-density one do expect Boltzmann-distribution for the momentum. The result for the averaged data is $q_{\text {momentum }}=0.999 \pm 0.001$ for random initial condi- 
tion and total energy $E / N=0.675$. We have similar results for the other three settings, so we can conclude that the momentum-distribution is Boltzmannian as expected. We got the same result for $40^{3}$ lattice as well.

\section{Euclidean SU(3) pure gauge theory with numerical results}

Our second model is the quantum SU(3) Yang-Mills theory. The action of the model in Euclidean formalism is the following:

$$
S_{Y M}=\frac{1}{4} \int d^{4} x F_{\mu \nu}^{a} F_{\mu v}^{a},
$$

where $F_{\mu v}(x)=-\mathfrak{i} g F_{\mu \nu}^{a}(x) T_{a}$ is the gluon field strength tensor, $T_{a}$ are the generators of the Liealgebra and $g$ is the coupling constant.

We use the Wilson-action for the lattice formulation of the theory:

$$
S[U]=\sum_{p} \beta\left(1-\frac{1}{N} \operatorname{Re} \operatorname{Tr} U_{p}\right),
$$

where $U(x+\mu, x)$ is the parallel transporter from lattice coordinate $x$ to $x+\mu$ and the plaquette variable corresponding to $x$ is $U_{p}=U(x, x+v) U(x+v, x+v+\mu) U(x+v+\mu, x+\mu) U(x+\mu, x)$. It is well-known that the Wilson-action corresponds to the continuum theory if one chooses $\beta=$ $2 N / g^{2}$ (in our case $N=3$ ) and the connection between the parallel transporters and the gauge fields is $U(x, \mu)=\mathrm{e}^{-a A_{\mu}(x)}$.

The local energy-density is now given by the plaquette energy:

$$
\varepsilon=\left\langle 1-\frac{1}{\operatorname{Tr} \Pi} \operatorname{Tr} U_{p}\right\rangle
$$

We use Monte Carlo simulation with the well-known heat-bath algorithm to determine the distribution of $\varepsilon$. Our results are presented on graph 5. Tsallis $f(x)=a x^{n}(1+(q-1) l x)^{\frac{1}{1-q}}$ and Boltzmann $g(x)=A x^{N} \mathrm{e}^{-B x}$ fits are applied to the numeric data. The time-like size of the lattice were $N_{t}=2$ and the space-like was $N_{s}^{3}=50^{3}$.

\section{Conclusions and outlook}

In this work we have determined the local energy-density histogram of the classical $\Phi^{4}$ and the quantum SU(3) Yang-Mills theory. We have found that the Boltzmann-distribution does not fit well. However, the Tsallis-distribution is a good fit, similarly to experimental data. In case of the classical $\Phi^{4}$ theory, a detailed analysis is performed and the $q$ parameter is in the order of the experimental values.

The $q$ parameter for the SU(3) theory is currently under investigation, with similar analysis as in the $\Phi^{4}$ theory. To accomplish this task, one needs to perform continuum limit and termodynamical limit analysis. Furthermore, usually one must perform renormalisation as the energy gains additive and multiplicative factors. However, the quantity in focus is not the value of the energydensity, but only the shape of the local energy-density distribution (i.e. the $q$ parameter) which we do not expect to depend on this. 


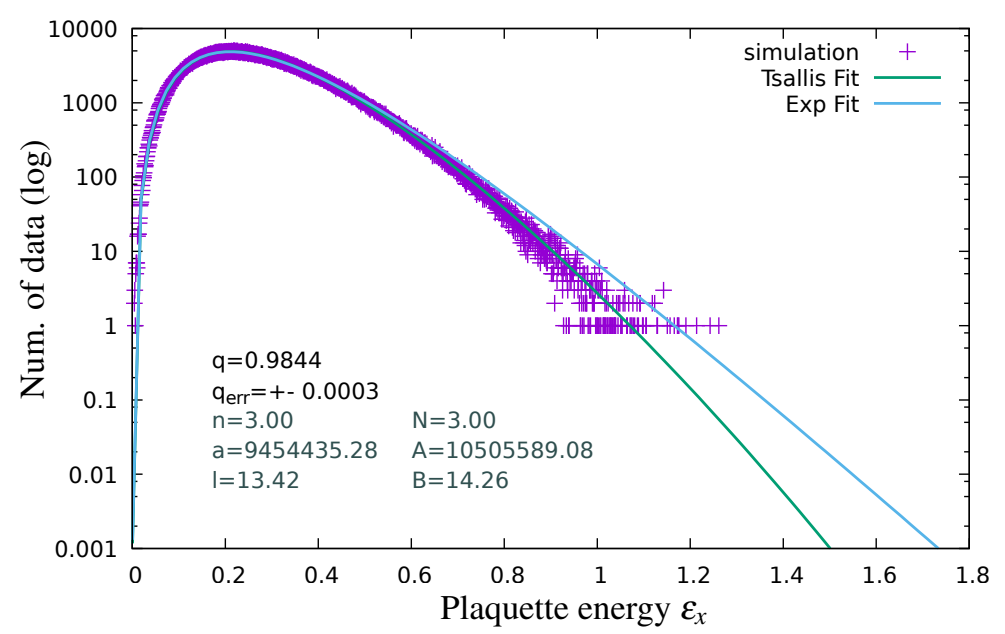

Figure 5: Plaquette energy histogram on semi-logscale after 20 heat-bath sweeps at $\beta=8, N_{t}=2, N_{s}=50$. The blue line is the Boltzmann and the green line is the Tsallis fit.

Our main goal is to perform similar analysis for QCD as well and to compare the results with experimental data.

This work was supported by the Hungarian Research Fund (OTKA) under contract No. K104292.

\section{References}

[1] E. Shuryak, Why does the quark gluon plasma at RHIC behave as a nearly ideal fluid? Prog. Part. Nucl. Phys. 53, 273 (2004) [hep-ph / 0312227$].$

[2] K. Aamodt et al. [ALICE Coll.], Production of pions, kaons and protons in pp collisions at $\sqrt{s}=900$ GeV with ALICE at the LHC, Eur.Phys.J. C71, 1655 (2011) [arXiv:1101.4110 [hep-ex] ]

[3] V. Khachatryan et al. [CMS Coll.], Transverse-momentum and pseudorapidity distributions of charged hadrons in pp collisions at $\sqrt{s}=7 \mathrm{TeV}$, Phys.Rev.Lett. 105, 022002 (2010), [arXiv:1005.3299 [hep-ex]]

[4] B.I. Abelev et al. [STAR Coll.], Strange particle production in $p+p$ collisions at $\sqrt{s}=200 \mathrm{GeV}$, Phys. Rev. C75, 064901 (2007), [nucl-ex/0607033]

[5] A. Adare et al. [PHENIX Coll.], Identified charged hadron production in $p+p$ collisions at $\sqrt{s}=200$ and 62.4 GeV, Phys. Rev. C83, 064903 (2011), [arXiv:1102.0753 [nucl-ex] ]

[6] J. Cleymans and D. Worku, The Tsallis Distribution in Proton-Proton Collisions at $\sqrt{s}=0.9 \mathrm{TeV}$ at the LHC, J.Phys. G39, 025006 (2012) [arXiv: 1110.5526 [hep-ph] ].

[7] V. Khachatryan et al. [CMS Coll.], Strange Particle Production in pp Collisions at $\sqrt{s}=0.9$ and $7 \mathrm{TeV}$, JHEP 05, 064 (2011), [arXiv: 1102.4282 [hep-ex] ]

[8] K. Urmossy, G.G. Barnafoldi, Sz. Harangozo, T.S. Biro and Z. Xu, A 'soft + hard' model for heavy-ion collisions, arXiv:1501.02352 [hep-ph], (2015)

[9] M.M. Homor and A. Jakovac, Shear viscosity of the $\Phi^{4}$ theory from classical simulation, Phys. Rev. D92, 105011 (2015), [arXiv: 1505.00930 [hep-th] ] 